\title{
Saline collection media and an extraction free workflow enables massively scalable, highly sensitive, and cost-effective SARS-CoV-2 testing
}

\author{
Yan Wei Lim \\ Helix \\ Nicole Leonetti \\ Helix \\ Aakash Amin \\ Helix \\ Henrique Machado \\ Helix \\ Natasha Bonilla \\ Helix \\ James Lu \\ Helix \\ K.David Becker ( $\nabla$ david.becker@helix.com ) \\ Helix
}

\section{Method Article}

Keywords: Extraction-Free, SARS-CoV-2, RT-qPCR assay, saline

Posted Date: November 13th, 2020

DOl: https://doi.org/10.21203/rs.3.rs-106189/v1

License: (c) (i) This work is licensed under a Creative Commons Attribution 4.0 International License. Read Full License 


\section{Abstract}

The challenges in scaling up SARS-CoV-2 testing capacity include shortages in the supply chain for consumables and reagents. Improvements in consumption patterns can be obtained through removal of key processing steps, including RNA extraction. Here, we present a scalable and validated extraction-free method for the detection of SARS-CoV-2 from swab specimens in saline, with a limit of detection at 1,000 $\mathrm{GCE} / \mathrm{mL}$ and a sensitivity and specificity of $100 \%$.

\section{Main Text}

With sufficient data that suggest SARS-CoV-2 can be spread by pre-symptomatic/asymptomatic carriers ${ }^{1}$, large-scale and repeated testing for SARS-CoV-2 infection is critical to control the spread of COVID-192. The standard workflow often includes total RNA extraction followed by real-time reverse transcriptase quantitative polymerase chain reaction (RT-qPCR). One of the challenges in scaling up SARS-CoV-2 testing capacity includes shortages in the supply chain for consumables and reagents. Therefore, reduced consumption can be achieved through removal of key processing steps such as RNA extraction.

An extraction-free workflow is less laborious than a RNA extraction workflow, but it is also less robust and prone to failure due to reaction inhibition by specimen components ${ }^{3}$. Studies suggested that dry swabs 4,5 and swab samples stored in appropriate transport media such as Universal/Viral Transport Media (UTM/VTM $)^{3,5-10}$ or water ${ }^{6}$ can be tested by RT-qPCR without the need for RNA extraction. The general workflow includes a lysis step, incubating samples between $70^{\circ} \mathrm{C}$ to $99^{\circ} \mathrm{C}$ for $5-15$ minutes, followed by RT-qPCR setup using a small amount of sample and specific RT-qPCR mastermixes. Only one of these studies $^{8}$ reported a limit of detection (LoD), which is greater than 6,000 genomic copies equivalent $/ \mathrm{mL}$ $(\mathrm{GCE} / \mathrm{mL})$ and it was high for a PCR-based assay ${ }^{11}$. In addition, the increased demand for testing has constrained the supply of UTM/VTM. Alternative transport media and, at the very least, an end-to-end protocol with comparable sensitivity to extraction-based methods is essential to alleviating possible trade-offs between efficiency and sensitivity.

Saline is easily obtainable and most importantly has demonstrated stability and usability in swab-based sampling ${ }^{12}$. Saline transport media is a mixture of salt (sodium chloride) and water at a pH of 4.5-7.0, which is similar to the sodium concentration of human bodily fluids. Saline has been shown to perform poorly in an extraction-free workflow when the sample is added directly into the RT-qPCR reaction ${ }^{3,6}$. However, the workflow described here was adapted from a saliva-based SARS-CoV-2 molecular test ${ }^{8}$ at which the sample is first diluted with 1X TBE ((Tris/Borate/EDTA Buffer) and then treated with heat at $95^{\circ} \mathrm{C}$ for 15 minutes. The heating step lysed the viral particles, and is also likely to inactivate the 
inhibitory components in the sample, therefore allowing better sensitivity in detecting SARS-CoV-2 nucleic acid. The method is further optimized to be used for processing saline-based swab specimens.

To establish the limit of detection (LoD) of the assay, contrived samples were generated from upper respiratory specimens negative for SARS-CoV-2 collected via anterior nares swab in $0.85 \%$ saline solution. The samples were pooled to obtain a large volume of negative matrix and spiked with gammairradiated 2019 SARS-CoV-2 virus (BEI Resources) at an appropriate concentration. Triplicates were screened at each concentration of inactive virus, ranging from $4,000 \mathrm{GCE} / \mathrm{mL}$ to $250 \mathrm{GCE} / \mathrm{mL}$ (Figure 1). The final LoD was confirmed using 20 replicates. All three replicates were called positive at $500 \mathrm{GCE} / \mathrm{mL}$ during LoD screening; however, only 16/20 samples were called positive at $500 \mathrm{GCE} / \mathrm{mL}$ during confirmation. The extraction-free LoD was confirmed at $1,000 \mathrm{GCE} / \mathrm{mL}$ with $20 / 20$ positive samples.

To evaluate the performance of extraction-free assay on clinical samples, this study used 30 positive and 30 negative remnant clinical swab samples in saline provided by an independent clinical lab. The samples were processed through the Helix standard extraction workflow as well as the extraction-free workflow. Cycle quantification $(\mathrm{Cq})$ was used to measure viral load and guide the qualitative interpretation of samples. The extraction-free workflow achieved $100 \%$ concordance with clinical samples tested with extraction workflow. However, the median Cq of the extraction-free workflow was $\sim 3-4 \mathrm{Cq}$ higher compared to the extraction workflow (Figure 2a). There is a linear correlation of the Cq between the extraction and $\mathrm{EF}$ workflows with the exception of two low positive samples on $\mathrm{N}$ gene amplification (Figure 2b).

Here, we presented a massively scalable, highly sensitive and cost effective method in detecting SARSCoV-2 for SARS-CoV-2 testing using saline collection media. The method outlined here consists of a minimal number of steps and utilizes a standard qPCR assay downstream, thus allowing direct implementation into the existing workflow. Saline has demonstrated usability in swab-based sampling ${ }^{12}$ and is easily obtainable. Saline can be stored at room temperature. Upon sample collection, the sample is stable for up to 54 hours without special storage conditions ${ }^{12}$. However, saline-based specimens have been shown to perform poorly in extraction-free workflow ${ }^{3,6}$. In contrast, the method presented here utilizes TBE dilution, heat treatment, and large sample input volumes to achieve $100 \%$ sensitivity and specificity in 60 clinical samples, and a LoD at 1,000 GCE/mL, which is comparable to assays using extraction-based methods ${ }^{11,13}$. The limitations of this study include the relatively small number of available clinical samples that preclude a more thorough analysis of sensitivity compared to extractionbased workflows. Even though the Cqs from the extraction-free workflow are higher than using extracted RNA, the LoD of the extraction-free workflow is equivalent, and detection of SARS-CoV-2 in clinical samples is highly correlated between the two methods. Our results suggest that a properly validated 
extraction-free RT-qPCR workflow can achieve the level of accuracy and sensitivity needed for reliable detection of SARS-CoV-2 in clinical samples. The extraction-free workflow using saline transport media removes supply chain constraints, has high accuracy and sensitivity, and it is simple, cost effective and massively scalable.

\section{References}

1. Furukawa, N. W., Brooks, J. T. \& Sobel, J. Evidence Supporting Transmission of Severe Acute Respiratory Syndrome Coronavirus 2 While Presymptomatic or Asymptomatic - Volume 26, Number 7 -July 2020 - Emerging Infectious Diseases journal - CDC. doi:10.3201/eid2607.201595.

2. Taipale, J., Romer, P. \& Linnarsson, S. Population-scale testing can suppress the spread of COVID-19. medRxiv 2020.04.27.20078329 (2020) doi:10.1101/2020.04.27.20078329.

3. Smyrlaki, I. et al. Massive and rapid COVID-19 testing is feasible by extraction-free SARS-CoV-2 RTPCR. Nat. Commun. 11, 4812 (2020).

4. Srivatsan, S. et al. Preliminary support for a "dry swab, extraction free" protocol for SARS-CoV-2 testing via RT-qPCR. bioRxiv 2020.04.22.056283 (2020) doi:10.1101/2020.04.22.056283.

5. Brown, J. R., Atkinson, L., Shah, D. \& Harris, K. Validation of an extraction-free RT-PCR protocol for detection of SARS-CoV2 RNA. medRxiv 2020.04.29.20085910 (2020) doi:10.1101/2020.04.29.20085910.

6. Merindol, N. et al. SARS-CoV-2 detection by direct rRT-PCR without RNA extraction. J. Clin. Virol. 128, 104423 (2020).

7. Bruce, E. A. et al. DIRECT RT-qPCR DETECTION OF SARS-CoV-2 RNA FROM PATIENT NASOPHARYNGEAL SWABS WITHOUT AN RNA EXTRACTION STEP. bioRxiv 2020.03.20.001008 (2020) doi:10.1101/2020.03.20.001008.

8. Hasan, M. R. et al. Detection of SARS-CoV-2 RNA by direct RT-qPCR on nasopharyngeal specimens without extraction of viral RNA. PLOS ONE 15, e0236564 (2020).

9. Grant, P. R., Turner, M. A., Shin, G. Y., Nastouli, E. \& Levett, L. J. Extraction-free COVID-19 (SARS-CoV-2) diagnosis by RT-PCR to increase capacity for national testing programmes during a pandemic. bioRxiv 2020.04.06.028316 (2020) doi:10.1101/2020.04.06.028316.

10. Beltrán-Pavez, C. et al. SARS-CoV-2 detection from nasopharyngeal swab samples without RNA extraction. bioRxiv 2020.03.28.013508 (2020) doi:10.1101/2020.03.28.013508.

11. Arnaout, R. et al. SARS-CoV2 Testing: The Limit of Detection Matters. bioRxiv (2020) doi:10.1101/2020.06.02.131144.

12. Everlywell COVID-19 test Home Collection Kit EUA Summary.

13. CDC 2019-Novel Coronavirus (2019-nCoV) Real-Time RT-PCR Diagnostic Panel.

14. Ranoa, D. R. E. et al. Saliva-Based Molecular Testing for SARS-CoV-2 that Bypasses RNA Extraction. bioRxiv 2020.06.18.159434 (2020) doi:10.1101/2020.06.18.159434. 


\section{Declarations}

\section{Acknowledgement}

We thank Matthew Haynes and Stephen Riffle for editing the manuscript. We thank Laboratory Corporation of America for providing the remnants clinical samples. This work was partially supported by the National Institute of Health $(\mathrm{NIH})$ through the Rapid Acceleration of Diagnostics (RADX) grant.

\section{Conflict of Interest Disclosures}

YWL, NAL, AJA, HM, NB, JTL, DB are employees of Helix

\section{Online Only Methods}

\section{Specimen Collection Materials}

Oropharyngeal Flocked Swab (Affordable IHI) was used in this study. $0.85 \%-0.90 \%$ saline was used as transport media.

\section{Sample Preparation for Limit of Detection and Confirmation Studies}

Contrived samples were generated from upper respiratory specimens negative for SARS-CoV-2 collected via anterior nares swab in $0.85 \%$ saline solution. The samples were pooled to obtain a large volume of negative matrix. Gamma-irradiated 2019 SARS-CoV-2 virus (BEI Resources) was then spiked into the negative matrix at an appropriate concentration and treated at $65^{\circ} \mathrm{C}$ for 10 minutes. After the deactivation step, $50 \mu \mathrm{l}$ of the contrived sample was added to $2 \mathrm{X}$ volume $(100 \mu \mathrm{l})$ of 1 X TBE buffer (method adopted from Ranoa et. al. ${ }^{14}$ ) with 1:200 diluted MS2 internal control (Thermo Fisher, PN A47814), corresponding to a final MS2 dilution of 1:300 from original stock. Diluted samples were treated at $95^{\circ} \mathrm{C}$ for 15 minutes prior to RT-qPCR.

\section{Sample preparation for clinical samples}

A total of 30 positive for SARS-CoV-2 and 30 negative clinical remnant samples derived from anterior nares swabs collected in normal saline were provided by an independent clinical lab (Laboratory Corporation of America). The samples were stored at $-80^{\circ} \mathrm{C}$ upon initial processing, and were shipped in dry ice to the Helix laboratory. Samples were heat-inactivated at $65^{\circ} \mathrm{C}$ for $10 \mathrm{~min}$ upon receiving. The samples were processed through the Helix COVID-19 Test (EUA2016360), as well as the extraction-free (EF) workflow. A total of $50 \mu \mathrm{l}$ of each sample was added to $100 \mu \mathrm{l}$ of 1X TBE with MS2 internal control 
(1:300 MS2 final dilution of original stock). For each sample, $14.25 \mu \mathrm{l}$ was transferred to a new Hard-Shell 384-well PCR plate for lysis at $95^{\circ} \mathrm{C}$ for 15 minutes. Per sample, $5 \mu$ of One-step RT-PCR mastermix MDX016 (Meridian BioScience) and $0.75 \mu$ of primer/probe mix from TaqPath ${ }^{\text {TM }}$ COVID-19 Combo Kit (Thermo Fisher) was added directly to the lysed samples, followed by RT-qPCR at a $20 \mu$ total reaction volume.

\section{RT-PCR setup for extraction-free workflow}

The RT-qPCR assay was set up using the TaqPath ${ }^{\text {TM }}$ COVID-19 Combo Kit (Thermo Fisher) from Thermo Fisher (EUA200010) and Inhibitor Tolerant 1-Step RT-qPCR Mastermix MDX016 (Meridian BioScience). The cycling conditions are shown in Table 1. The RT-qPCR assay was carried out with the QuantStudio 7 Flex Real-Time PCR System.

Table 1: Temperature and cycling conditions for Helix's extraction-free workflow using Meridian MDX016 mastermix.

\begin{tabular}{|llll|}
\hline Meridian MDX016 One Step RT qPCR 4X Mastermix & Temperature & Time & Cycles \\
\hline RT reaction & $52^{\circ} \mathrm{C}$ & $20 \mathrm{~min}$ & 1 \\
\hline Enzyme activation & $95^{\circ} \mathrm{C}$ & $2 \mathrm{~min}$ & 1 \\
\hline Denature & $95^{\circ} \mathrm{C}$ & $5 \mathrm{sec}$ & 45 \\
\cline { 1 - 2 }${ }^{*}$ Anneal/Extend (*Read) & $62^{\circ} \mathrm{C}$ & $60 \mathrm{sec}$ & \\
\hline
\end{tabular}

Interpretation of Sample based on the number of targets amplified

The targeted gene is considered positive if the amplification curve crosses the threshold line within 39 quantification cycles $(\mathrm{Cq}<39)$ and has a $\mathrm{Cq}$ confidence score $>0.8$. The final outcome of the individual sample is based on the number of targets detected as shown in Table 2. Cycle quantification (Cq) was used for qualitative interpretation of samples. True positives (TP) were defined by test results generated using the Helix COVID-19 Test. Sensitivity was defined as (TP)/(TP+FN) and specificity was defined as (TN)/(TN+FP).

Table 2: Interpretation of the RT-qPCR results per sample. 


\begin{tabular}{|llllll|}
\hline ORF1ab & N gene & S gene & MS2 & Status & Results \\
\hline NEG & NEG & NEG & NEG & Invalid & NA \\
\hline NEG & NEG & NEG & POS & Valid & SARS-CoV-2 not detected \\
\hline NEG & NEG & $\begin{array}{l}\text { POS with } \\
\text { Cq }>37^{*}\end{array}$ & POS & Valid & SARS-CoV-2 not detected \\
\hline Tnly one SARS-CoV-2 target $=$ POS & POS or NEG & Valid & SARS-CoV-2 Inconclusive \\
\hline
\end{tabular}

* Criteria to exclude artifacts introduced by low level $\mathrm{S}$ gene amplification in negative samples, POS with $\mathrm{Cq}>37$ and $\mathrm{Cq}$ confidence score $>0.8$.

Statistical analysis

Differences between extraction and extraction-free Cqs of the clinical positive samples were analyzed using a paired t-test. Correlations between the clinical positive samples extraction and extraction-free Cqs per gene target were analyzed using Spearman's rank test. Analyses were performed with GraphPad Prism.

\section{Figures}


(a)

(b)

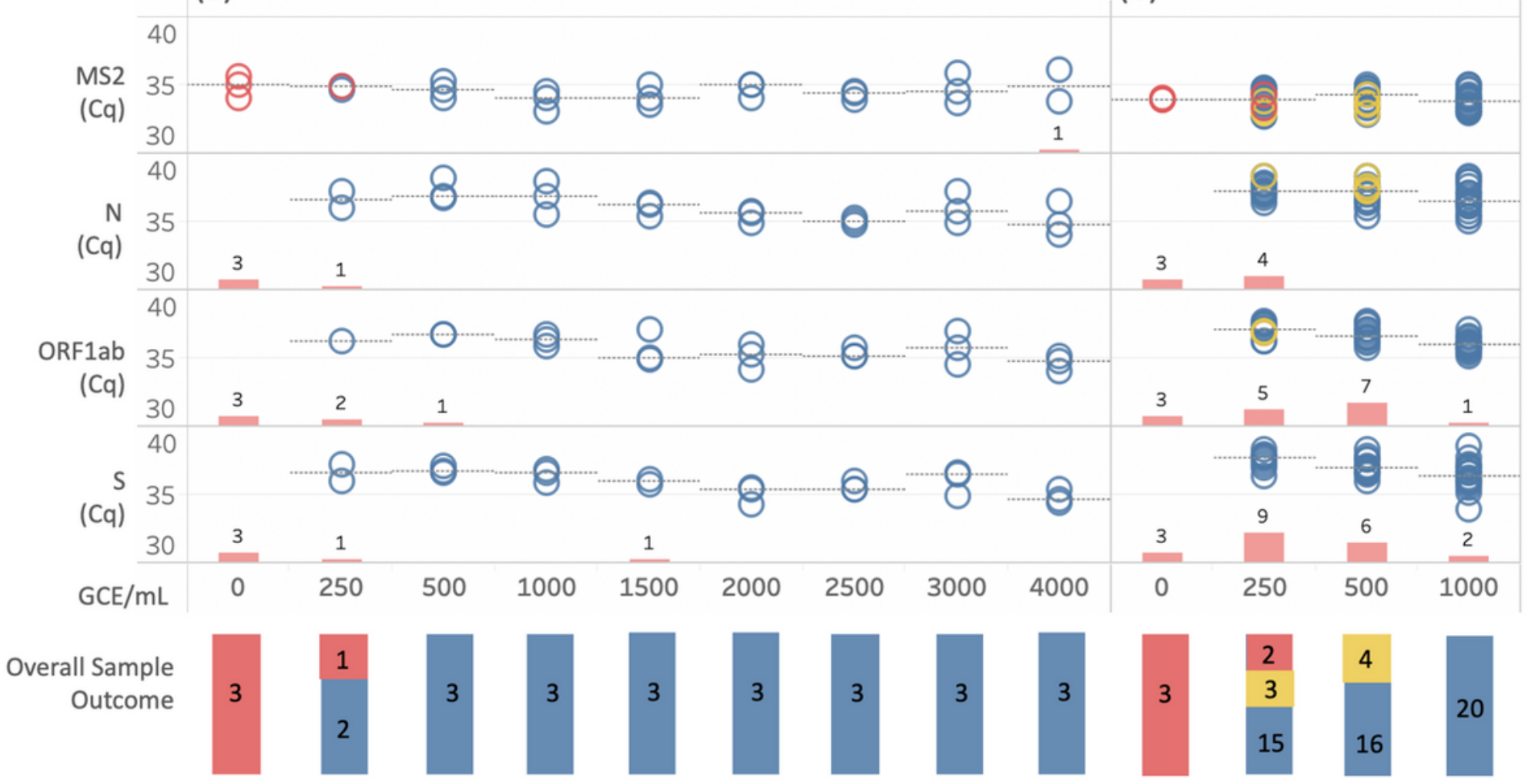

Figure 1

LoD of SARS-CoV-2 from anterior nares swab in saline solution. Gamma-irradiated SARS-CoV-2 was spiked into SARS-CoV-2 negative matrix. Specimens were mixed at 1:2 sample to TBE (Tris/Borate/EDTA Buffer) ratio, and were treated at $95^{\circ} \mathrm{C}$ for 15 min prior to RT-qPCR. All samples were analyzed in triplicate for LoD screening (a) and 20 replicates for LoD confirmation (b). Total non-amplified targeted calls are shown as pink bars. The LoD was confirmed by the amplification of $20 / 20$ replicates at $1,000 \mathrm{GCE} / \mathrm{mL}$. Blue $=$ Positive, Yellow $=$ Inconclusive, Red $=$ Not Detected . 


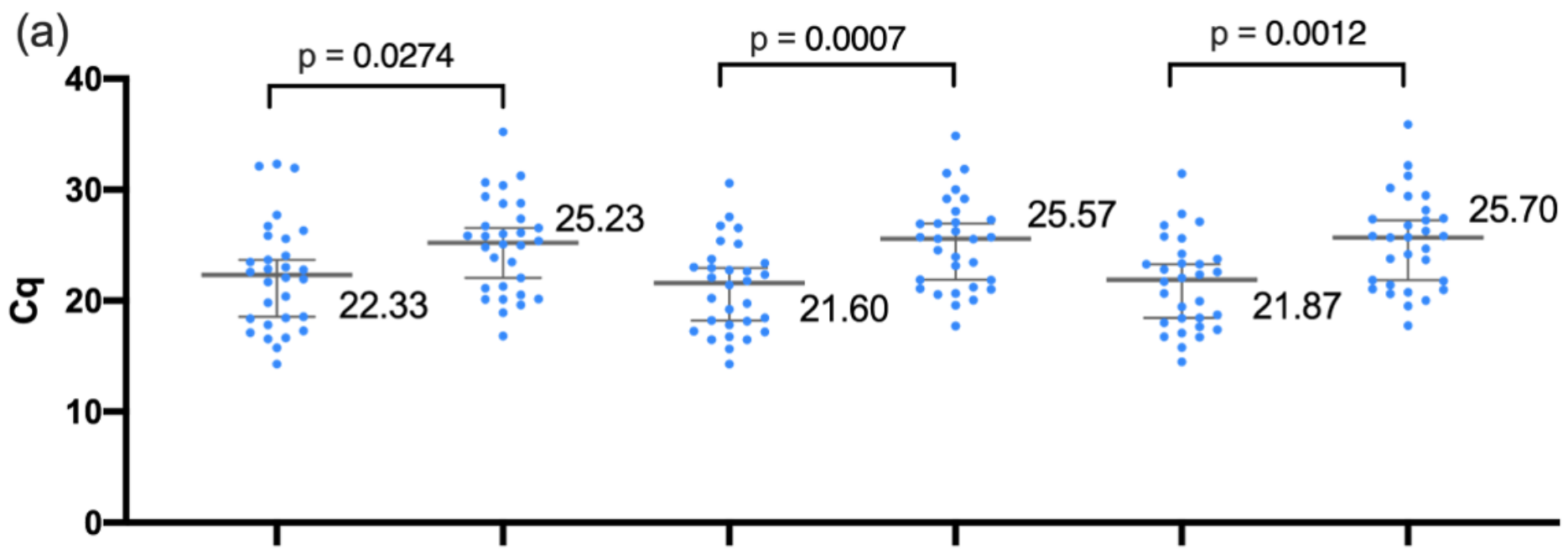

Extraction Extraction-Free Extraction Extraction-Free Extraction Extraction-Free

$\mathrm{N}$ gene

ORF1ab gene

S gene

(b)
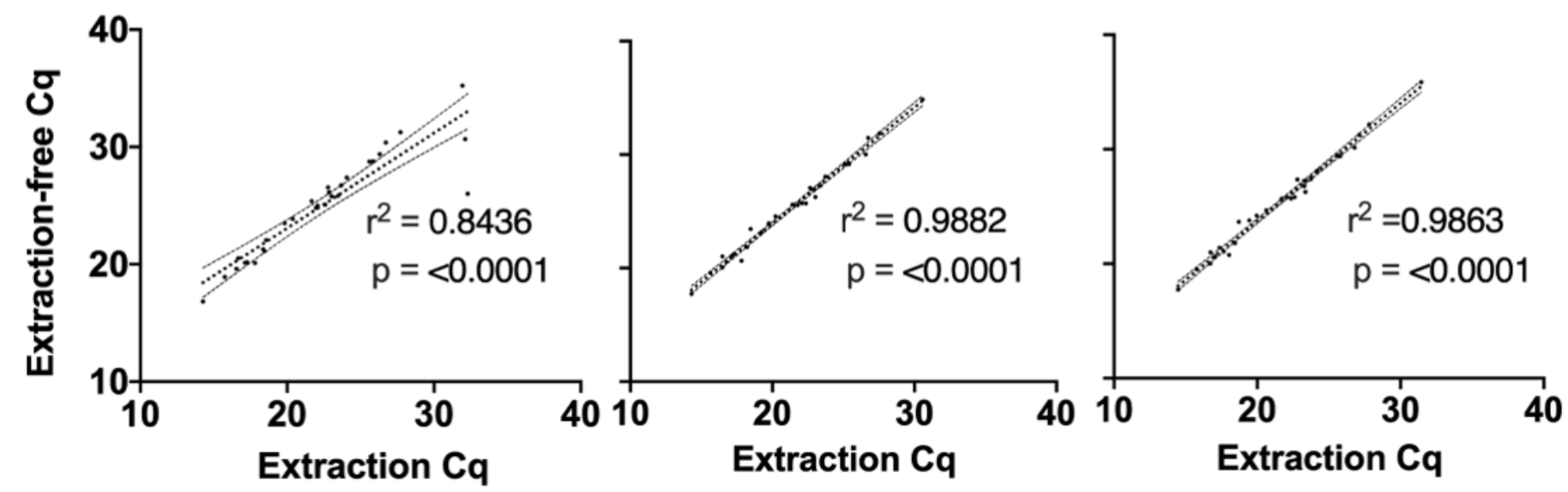

Figure 2

Comparison of $\mathrm{Cq}$ between extraction and extraction-free workflow across the same SARS-CoV-2 positive clinical samples. Distribution of $\mathrm{Cq}(\mathrm{a})$ and pairwise correlation (b) of $\mathrm{Cq}$ across the samples processed with extraction and extraction-free workflow. 